\title{
Determinantes sociais do desmame: contribuiçóes das diferentes abordagens metodológicas
}

\section{| ${ }^{1}$ Alessandra Rivero Hernandez, ${ }^{2}$ Celina Valderez Feijó Köhler I}

Resumo: O objetivo deste trabalho foi estudar as contribuições dos métodos quantitativos e qualitativos para a construção do conhecimento acerca dos determinantes sociais do processo de desmame, através da comparação entre os achados de pesquisas publicadas entre 2003 e 2009 na base de dados do sistema LILACS. Os determinantes sociais identificados nas investigações quantitativas foram expressos por variáveis de risco, enquanto os identificados nas investigações qualitativas deram visibilidade aos fatores intersubjetivos e objetivos que estruturam a ação social. O entendimento de que quantidade e qualidade são características que compõem os fenômenos complexos leva a propor a triangulação de métodos como uma estratégia para ampliar o conhecimento já existente acerca dos determinantes sociais do processo de desmame, e, assim, poder contribuir para a ampliação do período de amamentação.

> Palavras-chave: desmame; aleitamento materno; metodologia.
${ }^{1}$ Mestre em Saúde da Criança e do Adolescente pela Faculdade de Medicina da Universidade Federal do Rio Grande do Sul (UFRGS). Endereço eletrônico: riverohernandez@hotmail.com

${ }^{2}$ Mestre em Enfermagem EENF pela Universidade Federal Do Rio Grande do Sul (UFRGS). Endereço eletrônico: celinakohler@saude.rs.gov.br 
A amamentação apresenta profusos benefícios (TOMA; REA, 2008), bem conhecidos pela comunidade científica e difundidos na sociedade, principalmente aqueles que dizem respeito à criança. Mesmo com essa ampla divulgação, as durações tanto do aleitamento materno exclusivo quanto do aleitamento materno no Brasil estão muito aquém do período recomendado pela Organização Mundial da Saúde e pelo Ministério da Saúde (BRASIL, 2005), a saber: aleitamento materno exclusivo nos seis primeiros meses e complementado com outros alimentos por dois anos ou mais. As medianas de aleitamento materno exclusivo e de aleitamento materno no conjunto das capitais brasileiras e Distrito Federal, em 2008, foram de 54,1 dias (1,8 meses) e 341,6 dias (11,2 meses), respectivamente (BRASIL, 2009). Diante do exposto, muitos pesquisadores têm se dedicado a investigar as razões do processo de desmame em consonância com a perspectiva de que a amamentação é um fenômeno híbrido entre a natureza e a cultura, no qual os determinantes sociais tendem a preponderar diante daqueles de caráter biológico (ALMEIDA, 1999).

$\mathrm{Na}$ produção do conhecimento sobre os determinantes sociais do processo de desmame, os pesquisadores têm utilizado tanto métodos quantitativos como qualitativos. O método quantitativo rege-se pelos pressupostos da epidemiologia, a qual estuda a distribuição das doenças e suas causas em populações humanas por meio da quantificação, usando, para isso, cálculos matemáticos e técnicas estatísticas de amostragem e análise. O objeto da epidemiologia é de natureza probabilística (ALMEIDA FILHO; ROUQUAYROL, 2006). Minayo (2008a, p.21) refere que o método qualitativo "trabalha com o universo dos significados, dos motivos, das aspirações, das crenças, dos valores e das atitudes". Seu objeto de estudo é o mundo das relações, das representações e da intencionalidade. Tais aspectos não podem ser mensurados através de cálculos estatísticos.

Para discutir essas metodologias, faz-se necessário distinguir as teorias que as fundamentam. A metodologia quantitativa apóia-se no Positivismo, o qual considera que: a realidade fundamenta-se em fatos passíveis de observação; o mundo social opera de acordo com leis causais; o conhecimento do dado deve ser objetivo, neutro e sem juízo de valor; e seus achados são capazes de fornecer generalizaçôes e regularidades. A metodologia qualitativa é sustentada 
por diferentes abordagens compreensivas que apresentam como aspectos em comum: o foco na vivência; o reconhecimento da complexidade das realidades humanas; o contato com as pessoas, que ocorre nos seus próprios contextos sociais; e o realce do encontro intersubjetivo entre a relação pesquisador e sujeitos investigados. Além disso, na metodologia qualitativa, os resultados são expressos como a racionalidade dos contextos e a lógica interna dos diversos atores e grupos em estudo. A realidade é vista de forma dinâmica, trazendo à tona o ponto de vista dos vários atores frente a um projeto social em constante construção. Mais ainda, as conclusões nesse tipo de método não podem ser universalizadas, mas o estudo aprofundado da realidade possibilita inferências mais abrangentes (MINAYO, 2008a; MINAYO, 2008b).

O desmame é definido como a interrupção do aleitamento materno. Entretanto, muitos trabalhos não se dedicam apenas a conhecer as causas do abandono total da prática da amamentação, mas observam também os determinantes relativos à suspensão precoce do aleitamento materno exclusivo. Nessa situação, a criança pode estar recebendo leite materno, independente de estar recebendo qualquer alimento ou líquido. Assim, optou-se neste estudo por utilizar a expressão "processo de desmame", a fim de incluir tanto os determinantes da interrupção precoce do aleitamento materno exclusivo como os do aleitamento materno.

No presente trabalho estudaram-se as contribuições dos métodos quantitativos e qualitativos para a construção do conhecimento acerca dos determinantes sociais do processo de desmame, através da comparação entre os achados de pesquisas que utilizaram essas abordagens.

\section{Método}

Realizou-se um levantamento dos artigos publicados entre 2003 e 2009 da base de dados do sistema LILACS (Literatura Latino-Americana e do Caribe em Ciências da Saúde), utilizando as palavras-chave desmame, aleitamento materno e amamentação, identificando-se 112 trabalhos. Destes, selecionou-se aqueles cujo resumo demonstrasse estudar as causas do processo de desmame. Foram excluídas as dissertações e teses, os artigos cujo texto completo não foi possível localizar, e os artigos que utilizaram dados secundários. Assim, permaneceram 36 artigos, sendo 27 de abordagem quantitativa e 9 de abordagem qualitativa (tabela 1). 

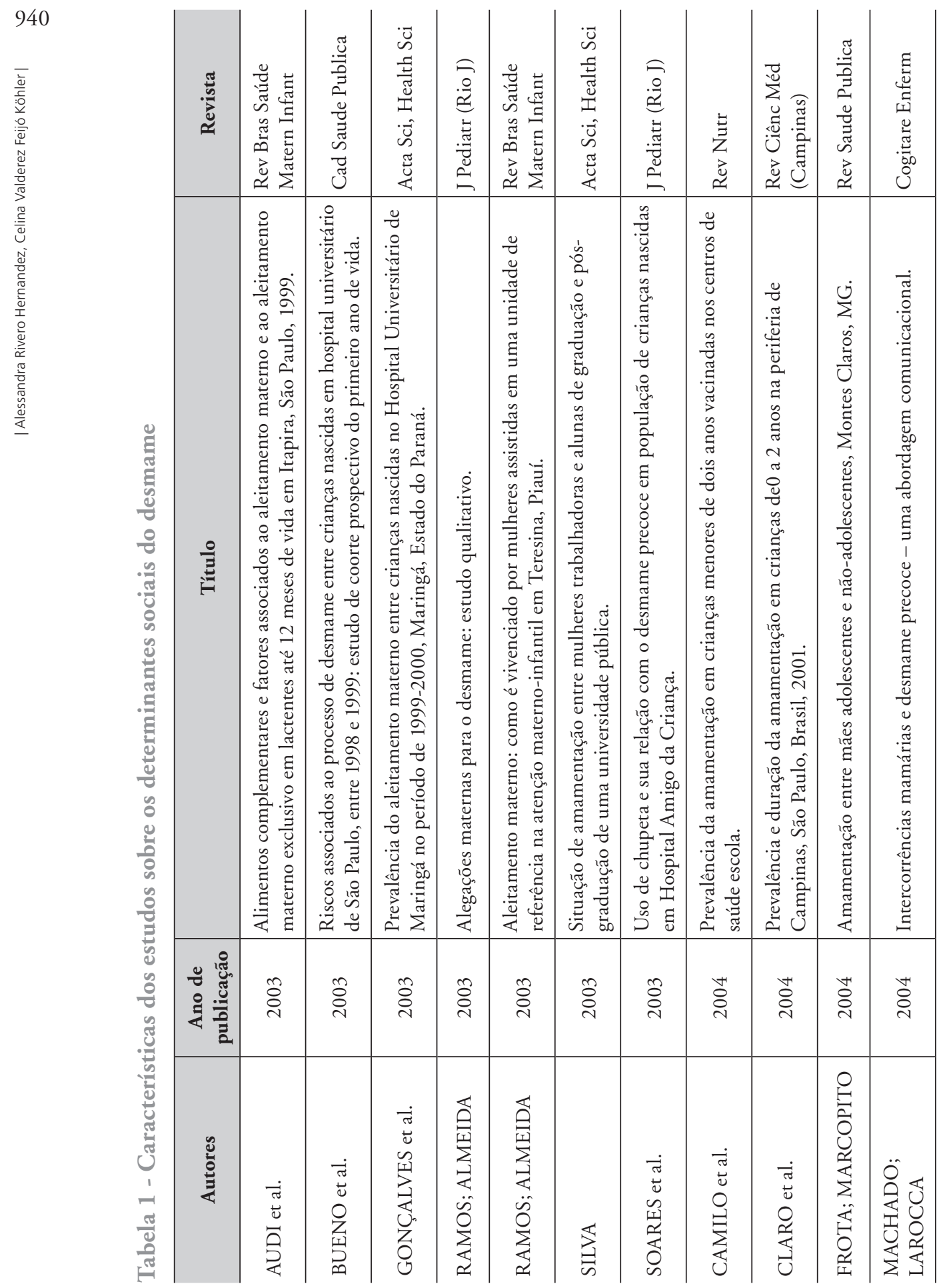


\begin{tabular}{|c|c|c|c|c|c|c|c|c|c|c|c|c|}
\hline 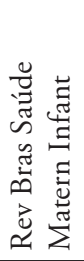 & 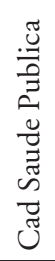 & 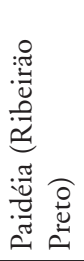 & 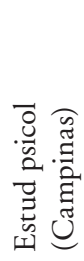 & 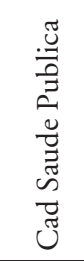 & 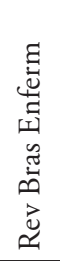 & 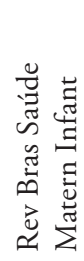 & 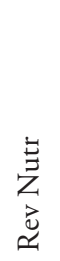 & 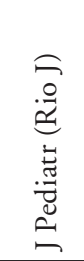 & 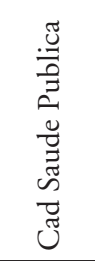 & 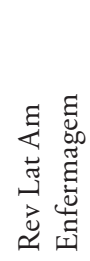 & 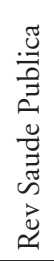 & 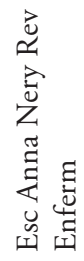 \\
\hline 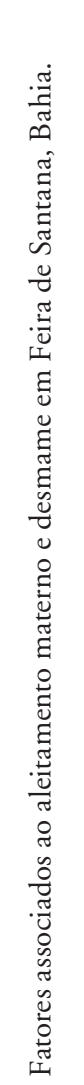 & 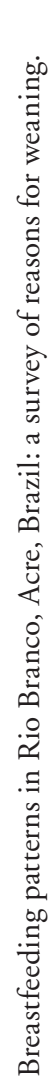 & 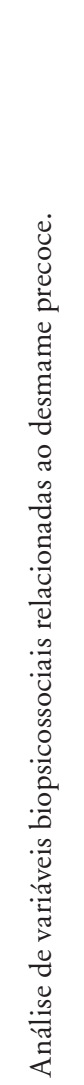 & 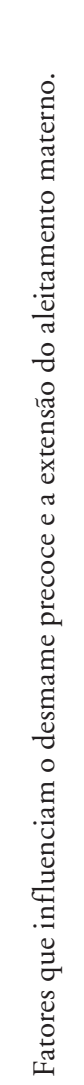 & 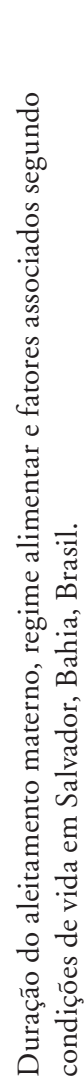 & 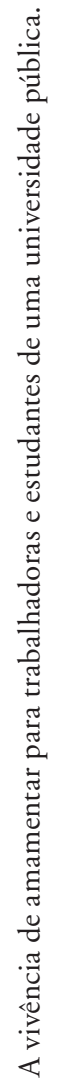 & 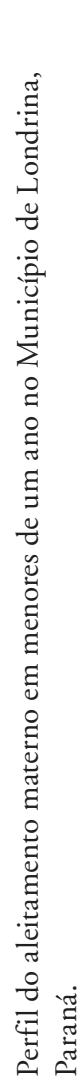 & 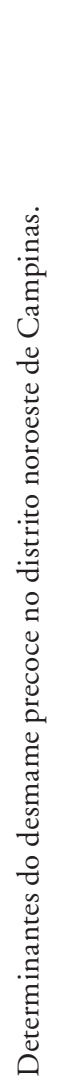 & 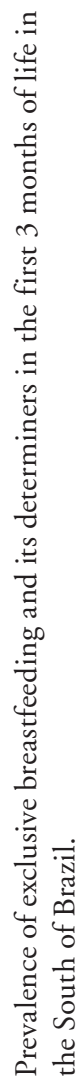 & 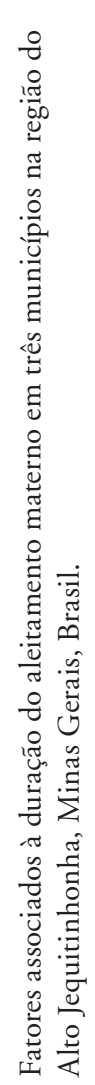 & 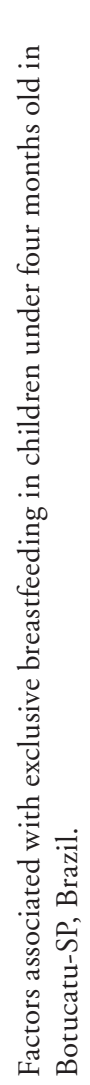 & 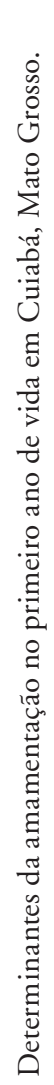 & 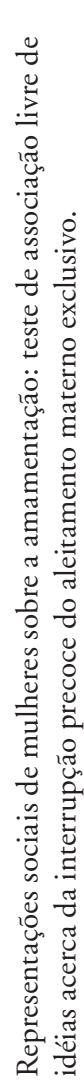 \\
\hline 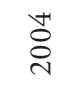 & $\begin{array}{l}\stackrel{+}{8} \\
\text { ¿ }\end{array}$ & $\begin{array}{l}\text { ¿̂ } \\
\stackrel{丶}{0}\end{array}$ & $\begin{array}{l}\text { ¿े } \\
\text { }\end{array}$ & 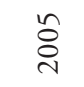 & 苂 & 占 & $\begin{array}{l}\text { ̊̊ } \\
\stackrel{\sim}{*}\end{array}$ & 号 & 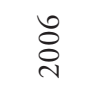 & 용 & ôे & 穴 \\
\hline 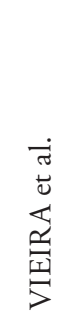 & 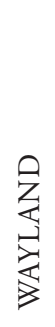 & 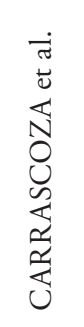 & 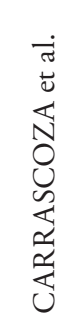 & 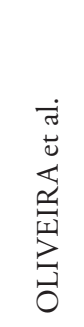 & $\underset{⿱ 乛}{\exists}$ & 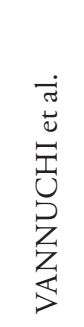 & 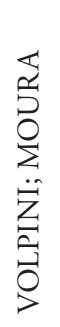 & 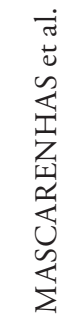 & 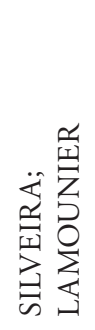 & 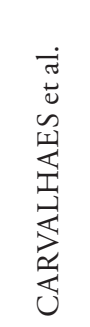 & 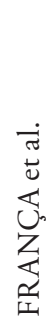 & 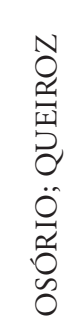 \\
\hline
\end{tabular}




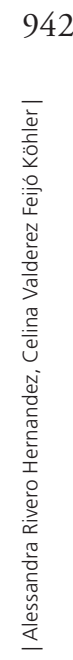

\begin{tabular}{|c|c|c|c|c|c|c|c|c|c|c|c|}
\hline 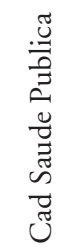 & 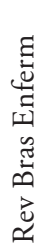 & 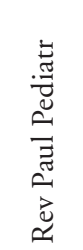 & 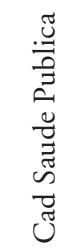 & 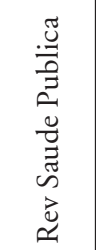 & 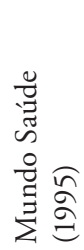 & 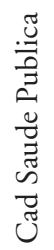 & 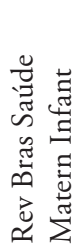 & 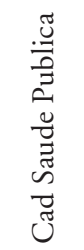 & 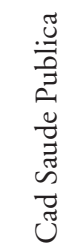 & 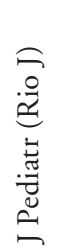 & 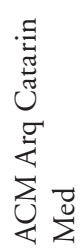 \\
\hline 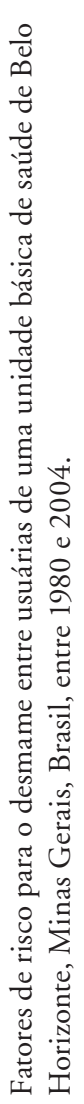 & 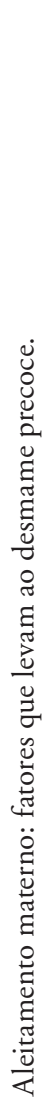 & 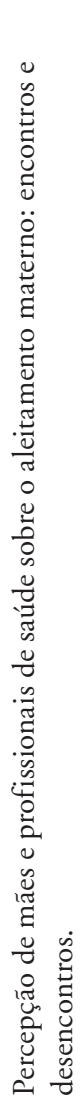 & 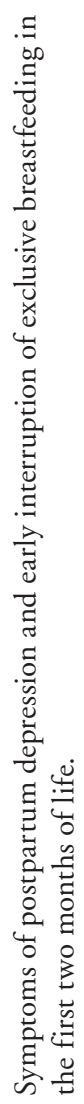 & 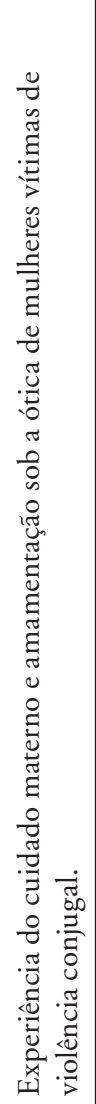 & 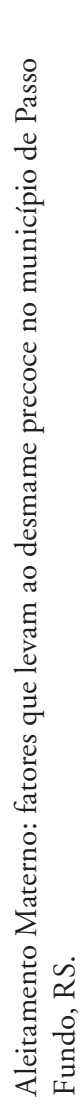 & 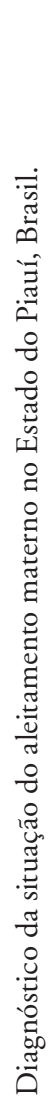 & 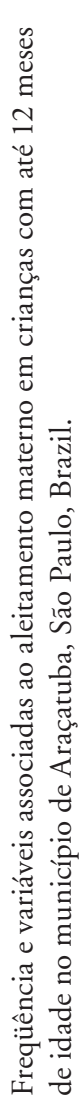 & 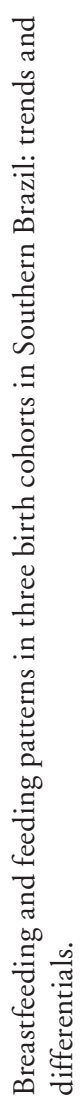 & 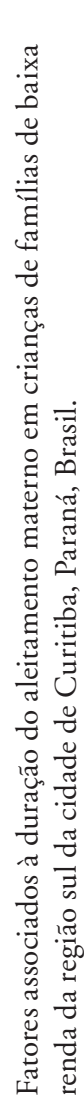 & 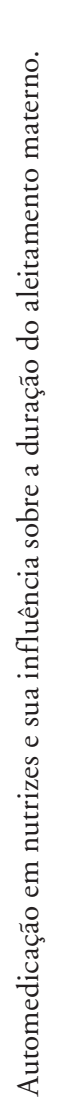 & 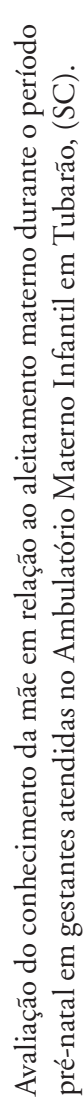 \\
\hline $\begin{array}{l}\infty \\
\stackrel{丶}{0}\end{array}$ & $\begin{array}{l}\infty \\
\stackrel{丶}{0}\end{array}$ & 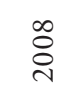 & $\stackrel{\infty}{\stackrel{8}{\circ}}$ & $\stackrel{\infty}{\stackrel{\infty}{\circ}}$ & $\begin{array}{l}\infty \\
\stackrel{丶}{\circ} \\
\stackrel{\sim}{\circ}\end{array}$ & $\begin{array}{l}\infty \\
\stackrel{\sim}{2} \\
\text { ㄱ. }\end{array}$ & $\begin{array}{l}\infty \\
\stackrel{2}{ } \\
\stackrel{2}{2}\end{array}$ & $\begin{array}{l}\infty \\
\stackrel{8}{0}\end{array}$ & & ஓे & 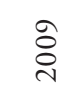 \\
\hline 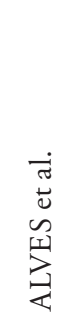 & 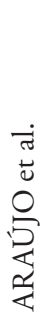 & 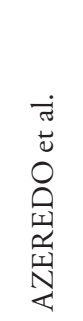 & 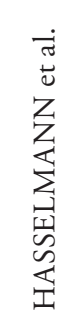 & 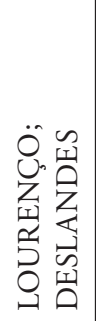 & 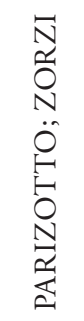 & 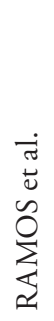 & 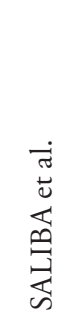 & 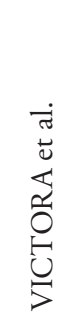 & 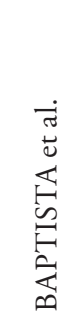 & 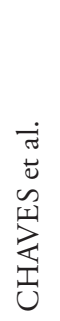 & 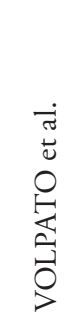 \\
\hline
\end{tabular}




\section{Artigos com abordagem quantitativa}

$\mathrm{O}$ determinante social que mais frequentemente se relacionou com a interrupção do aleitamento materno exclusivo e do aleitamento materno foi o uso de chupeta (VANNUCHI et al., 2005; SILVEIRA; LAMOUNIER, 2006; FRANÇA et al., 2007; RAMOS et al., 2008). Soares et al. (2003) observaram que a incidência de desmame até o sexto mês, em uma coorte de crianças amamentadas no primeiro mês, foi de 22,4\% para as crianças não usuárias de chupeta, e de 50,8\% para as usuárias $(\mathrm{p}<0,001)$. Mascarenhas et al. (2006) verificaram que os bebês que usaram chupeta tiveram um risco quatro vezes maior de não mamarem exclusivamente.

Quanto às características maternas, os trabalhos identificam como determinantes sociais para a interrupção, tanto do aleitamento materno exclusivo como para o aleitamento materno, a primiparidade e o trabalho (VANNUCHI et al., 2005; MASCARENHAS et al., 2006; FRANÇA et al., 2007; ALVES et al., 2008; BAPTISTA et al., 2009). Para o aleitamento materno exclusivo, encontraram-se como fatores de risco a idade precoce e menor escolaridade (BUENO et al., 2003; OLIVEIRA et al., 2005; FRANÇA et al., 2007), enquanto para o aleitamento materno, identificou-se o conceito de tempo ideal de aleitamento materno menor que seis meses (ALVES et al., 2008). Frota e Marcopito (2004) constataram que a adolescência apresentava um efeito diferente sobre o desmame conforme o estado conjugal: atuava como fator de proteção em mães adolescentes sem vida conjugal e como fator de risco naquelas com vida conjugal.

No que se refere às características paternas, verificam-se como determinantes sociais para o desmame: não residir com a criança (SILVEIRA; LAMOUNIER, 2006), não reconhecer os benefícios da amamentação para a criança, e ter opinião desfavorável, indiferente ou desconhecida sobre o aleitamento materno (ALVES et al., 2008). Enquanto Silveira e Lamounier (2006) observaram um risco maior de interrupção precoce do aleitamento materno se a escolaridade paterna fosse maior ou igual ao segundo grau completo, Mascarenhas et al. (2006) verificaram associação significativa entre escolaridade paterna menor que 5 anos e interrupção do aleitamento exclusivo antes dos três meses de vida.

Em relação às condições socioeconômicas da família, o estudo de Oliveira et al. (2005) identificou que precárias condições de vida, determinadas pelo índice de condiçôes de vida (ICV), estavam associadas à interrupção precoce do aleitamento 
exclusivo. Entre os achados de Mascarenhas et al. (2006), a renda familiar entre um e três salários mínimos apresentou associação significativa com interrupção do aleitamento exclusivo antes dos 3 meses de vida. Chama a atenção o fato de crianças de famílias com renda menor ou igual a um salário mínimo não apresentarem risco aumentado, o que, segundo os autores, poderia ser explicado pela falta absoluta de condições de adquirir o substituto para o leite materno dessas famílias. Ramos et al. (2008) verificaram que a prevalência de aleitamento materno foi menor entre as crianças menores de um ano cujas mães pertenciam às classes econômicas $\mathrm{A} e$ B. Victora et al. (2008) constataram que até 6-9 meses, o aleitamento materno era mais prevalente em famílias de renda mais alta, mas, depois dessa idade, passava a ser mais comum entre famílias mais pobres.

Quanto aos serviços de saúde, o acompanhamento em serviço público apresentou-se como fator de risco para interrupção tanto do aleitamento materno exclusivo como do aleitamento materno (VANNUCHI et al., 2005).

\section{Artigos com abordagem qualitativa}

Ao indagar as mulheres sobre as razões para o processo de desmame, as alegações mais comuns foram "leite fraco" ou "pouco leite" (RAMOS; ALMEIDA, 2003a; OSÓRIO; QUEIROZ, 2007). A análise das falas das entrevistadas permitiu que emergissem outros elementos interferentes no processo de amamentação como a falta de experiência materna; o fardo ocasionado pela amamentação; as relações com seus familiares, amigos e parceiros; o trabalho materno; a ambigüidade entre o querer e o poder amamentar, entre outras (RAMOS; ALMEIDA, 2003a; RAMOS; ALMEIDA, 2003b; SILVA, 2005; OSÓRIO; QUEIROZ, 2007).

Silva (2005), ao entrevistar trabalhadoras e estudantes de uma universidade, identificou a falta de estrutura da instituição (ausência de sala de ordenha apropriada, de material adequado para coleta e de refrigeração apropriada do leite) como condição que interfere profundamente na continuidade da amamentação. As entrevistadas se sentiram constrangidas em se expor, e inseguras em ofertar um leite potencialmente contaminado aos seus filhos. Após o retorno às atividades profissionais ou acadêmicas, com o passar do tempo, elas sentiram que seus colegas ou superiores foram "esquecendo" de suas necessidades diferenciadas para poder continuar amamentando. 
Ao entrevistar mulheres que iniciaram o processo de desmame, Ramos e Almeida (2003a) referem que veio à tona, invariavelmente, na fala das participantes, a solidão e o isolamento da mulher, bem como a necessidade de obter apoio para a efetivação dessa prática, tanto por parte do serviço de saúde como pelos outros segmentos da sociedade. Em outro estudo, os mesmos autores (RAMOS; ALMEIDA, 2003b), com o objetivo de ampliar a compreensão acerca de como as mulheres que desmamaram os seus filhos percebem a amamentação, destacam, através da análise do discurso das entrevistadas, a pouca efetividade na comunicação estabelecida entre as mulheres e os profissionais de saúde, e a incongruência entre as orientações recebidas no serviço de saúde e a prática vivida por elas.

\section{Discussão}

Os determinantes sociais apresentados nos estudos quantitativos associaram-se ao conceito de fatores de risco, central à epidemiologia moderna, ao expressarem um atributo (exposição) que aumenta a probabilidade de dar-se um determinado agravo (desfecho). Nos trabalhos de cunho qualitativo, os determinantes sociais surgiram como

injunções produzidas pelas crenças e vivências construídas no seio da família, dos vizinhos, do trabalho, da igreja, da política, da cultura, do sistema de saúde, entre outros, [que] são revalorizadas pelos usuários a partir de suas trajetórias de vida e redes de pertencimento (MARTINS, 2009b, p. 55).

Entre os artigos quantitativos analisados, o indivíduo é entendido como uma unidade estatística social, objetivado por determinantes sociais que produzem a sensação de serem realidades estanques e válidas para quaisquer situações (MARTINS, 2009a). Assim, nesses trabalhos observa-se amiúde a recomendação para que profissionais da saúde priorizem sua prática assistencial para gestantes e nutrizes consideradas de alto risco para o desmame. Contudo, analisando apenas os trabalhos de cunho quantitativo, há divergências que apontam a falta de confiança em extrapolar alguns determinantes sociais, observados na população investigada, para outras populações, como é o caso das variáveis de renda e de escolaridade do pai.

Por outro lado, nas investigaçôes qualitativas o indivíduo é percebido como uma unidade biopsíquica e cultural complexa que tem sua ação facilitada por fatores 
intersubjetivos e objetivos que estruturam a ação social (MARTINS, 2009a). Para Martins (2009a), as redes sociais são conectadas por mediadores humanos, tais como o cônjuge, a mãe, um familiar, uma vizinha, um profissional da saúde, e mediadores não-humanos, como é o caso das crenças e dos valores. Os mediadores podem tanto se apresentar como colaboradores, quando mediam conflitos e geram alianças e soluçóes, como inibidores, quando reproduzem conflitos. Essa complexa trama explicita a dificuldade em inferir os determinantes sociais identificados a nível populacional para os indivíduos, o que poderia resultar em uma grave falácia. A ocorrência da variável "mãe adolescente" tornar-se tanto em um fator de risco quanto de proteção, segundo seu estado matrimonial, sinaliza o emaranhado de relações que constitui o processo de desmame, relaçóes essas que os estudos qualitativos permitem emergir de modo mais espontâneo.

Nota-se, na abordagem quantitativa, o domínio do conhecimento científico do pesquisador sobre o conhecimento de senso comum do pesquisado. Há uma grande insistência na necessidade de explicar e convencer a mulher de que seu leite não é fraco ou em pouca quantidade, dos benefícios da amamentação e dos malefícios do uso da chupeta.

A figura do "leite fraco" foi criada pelos higienistas do século XIX, e atuava impondo a amamentação à mulher, responsabilizando-a e culpabilizando-a pelo desmame. Agiam unicamente na promoção do aleitamento materno, desconsiderando a necessidade de apoiar a mulher e ensiná-la como amamentar. $\mathrm{O}$ "leite fraco" surgiu para manter a salvo o higienismo da crise, já que uma grande variedade de exceções tornaria evidente sua incapacidade de solucionar as dificuldades presentes no ato de amamentar. Para a mulher, essa alternativa atenuava sua responsabilidade pelo desmame, razão de forte censura social (ALMEIDA, 1999).

Contrariamente, no método qualitativo, revela-se o conhecimento empírico do sujeito, adquirido no cotidiano por meio das experiências vivenciadas. Desse modo, percebe-se que convencer a nutriz de que seu leite não é fraco ou escasso pode não surtir efeito, uma vez que não se atua sobre os determinantes sociais que estão por trás dessa resposta socialmente aceita, ou seja, não há atuação sobre as dificuldades na técnica de amamentação e/ou a falta de apoio e solidariedade na rede social na qual está inserida. Esclarecer apenas que seu leite não é fraco faria com que o profissional da saúde atuasse como um mediador inibidor na teia 
social dessa mulher, o que reproduziria o conflito entre querer e poder amamentar (RAMOS; ALMEIDA, 2003a), ao invés de posicionar-se como um mediador colaborador, buscando identificar os reais problemas e mediar soluçôes.

Sem negar a importância da promoção do aleitamento materno, a prática profissional não poderia se limitar apenas a essa dimensão. Observa-se nos estudos quantitativos, por exemplo, que o uso da chupeta é muito frequente, mesmo quando as mulheres foram orientadas a não utilizá-la. As falas das mães, manifestas nos estudos qualitativos, permitem compreender a contradição existente entre as orientaçóes dos profissionais da saúde e a realidade vivida por elas, ao dar visibilidade aos sentimentos maternos de preocupação e de fardo quando a criança chora intensamente ou dorme pouco, uma vez que isso dificulta a realização de outras atividades de seu cotidiano (RAMOS; ALMEIDA, 2003b).

Será que simplesmente desaconsel har e enumerar todos os malefícios da utilização da chupeta não faz com que os profissionais atuem ainda no modelo higienista, informando a mãe para posteriormente responsabilizá-la e culpabilizá-la pelo uso desse utensílio? Não seria necessário também ouvi-la para refletir sobre a forma de amparar e apoiar essa mulher? Cabe lembrar também que se desconhece até então se a chupeta causa o desmame ou se é um marcador de dificuldades no aleitamento (VICTORA et al., 1997). Esse desconhecimento reforça a necessidade de operar sobre contextos mais amplos que possibilitem a consecução da amamentação e a renúncia ao uso da chupeta, pois mesmo que esse não seja um fator contribuinte para o desmame, permanece a ocorrência de outros malefícios, como as alterações nas funções orais decorrentes de sua utilização.

À medida que se desloca o olhar da sociedade em direção ao indivíduo, os fatores condicionantes da ação social vão se tornando mais complexos (MARTINS, 2009a). Nesse sentido, os resultados das pesquisas qualitativas vêm ao encontro do pensamento complexo, que propõe uma nova teoria interpretativa da realidade, na qual o fenômeno é visto como um emaranhado de relações. É por meio das interações, das relações, que ocorre a emergência das propriedades, ou seja, novas propriedades surgem a partir da organização das partes constituintes e que delas estão ausentes quando analisadas isoladamente. Os sistemas complexos apresentam um comportamento imprevisível, não-determinístico, caótico,

no entanto, isso não significa que não se possam fazer previsões muito precisas, mas elas se referem às características qualitativas do comportamento do sistema e não aos valores precisos de suas variáveis num determinado instante (CAPRA, 2006, p.116). 
com a organização de conteúdos aritmeticamente ordenados e determinísticos, termina negligenciando justamente os elementos não-causais, dissipativos e mesmo caóticos que estão presentes em todos os planos da vida social (MARTINS, 2009b, p.53).

Tanto o método qualitativo como o quantitativo têm recebido críticas. Enquanto o primeiro é desaprovado sobretudo por seu empirismo, pela subjetividade e pelo envolvimento emocional do pesquisador, o segundo é censurado especialmente por simplificar a complexidade da vida social, por limitar-se aos fenômenos observáveis e que podem ser quantificados e pela pretensa neutralidade dos investigadores (MINAYO, 2008b).

Ao reconhecer que qualquer forma de conhecimento é aproximada, que ambos os métodos aos quais este artigo faz referência são incompletos, e que todos os fenômenos têm atributos numéricos e qualidades, percebe-se a necessidade de utilizar as duas abordagens metodológicas para buscar explicar e compreender os determinantes sociais de fenômenos complexos, como é o processo de desmame. A triangulação de métodos articula essas abordagens de forma complementar, valorizando o qualitativo e o quantitativo, sendo este compreendido como um indicador e uma parte da qualidade dos fenômenos (MINAYO, 2008b). BriceñoLeón (2003) propõe quatro modelos de triangulação desses métodos, brevemente apresentados aqui com a finalidade de ilustrar alguns dos cruzamentos possíveis: a investigação qualitativa seguida da quantitativa; a investigação quantitativa seguida da qualitativa; a execução paralela das investigaçoos quantitativa e qualitativa; e a utilização de ambas as abordagens alternadamente.

Cabe, por fim, ressaltar que as políticas públicas e a prática profissional para promoção, proteção e apoio à amamentação não poderiam fundamentarse unicamente nos determinantes sociais do processo de desmame identificados nas pesquisas de cunho quantitativo para definir quais as mulheres a receberem uma atenção priorizada. Isso incorreria no engano de desconsiderar o contexto, desviando o foco para variáveis tais como renda e educação materna, que são de difícil atuação profissional com o objetivo de modificá-las. Essas variáveis, denominadas como macrossociológicas, são utilizadas de forma eficaz na gestão de sistemas hierárquicos, mas insuficientes para explicar as causas da ação humana (MARTINS, 2009a; MARTINS, 2009b). 
Por outro lado, as pesquisas qualitativas manifestam as relações sociais, as crenças e os sentimentos presentes no cotidiano determinantes do processo de desmame e que podem ser trabalhadas pelos profissionais da saúde através do acolhimento, escuta, aconselhamento e apoio. Essas variáveis, denominadas como microssociológicas, são fundamentais para se compreender as práticas individuais e socioculturais de saúde (MARTINS, 2009a; MARTINS, 2009b). Entretanto, o entendimento de que quantidade e qualidade são características que compóem os fenômenos complexos leva a propor a triangulação de métodos como estratégia para ampliar o conhecimento já existente acerca dos determinantes sociais do processo de desmame e assim poder contribuir para a ampliação do período de amamentação.

\section{Referências}

ALMEIDA, J.A.G. Amamentação: um híbrido natureza-cultura. Rio de Janeiro: Fiocruz, 1999. $120 \mathrm{p}$.

ALMEIDA FILHO, N. de; ROUQUAYROL, M.Z. Introdução à epidemiologia. Rio de Janeiro: Guanabara Koogan, 2006. 282 p.

ALVES, C.R.L.; GOULART, E.M.A.; COLOSIMO, E.A. et al. Fatores de risco para o desmame entre usuárias de uma unidade básica de saúde de Belo Horizonte, Minas Gerais, Brasil, entre 1980 e 2004. Cad Saude Publica, v. 24, n. 6, p. 1355-1367, 2008.

ARAÚJO, O.D. de; CUNHA, A.L. da; LUSTOSA, L.R. et al. Aleitamento materno: fatores que levam ao desmame precoce. Rev Bras Enferm, v. 61, n. 4, p. 488-492, 2008.

AUDI, C.A.F.; CORRÊA, A.M.S.; LATORRE, M. do R.D. de O. Alimentos complementares e fatores associados ao aleitamento materno e ao aleitamento materno exclusivo em lactentes até 12 meses de vida em Itapira, São Paulo, 1999. Rev. bras. saúde matern. infant, v. 3, n. 1, p. 85-93, 2003.

AZEREDO, C.M.; MAIA, T. de M.; ROSA, T.C.A. et al. Percepção de mães e profissionais de saúde sobre o aleitamento materno: encontros e desencontros. Rev. paul. Pediatr, v. 26, n. 4, p. 336-344, 2008.

BAPTISTA, G.H.; ANDRADE, A.H.H.K.G. de; GIOLO, S.R. Fatores associados à duração do aleitamento materno em crianças de famílias de baixa renda da regiāo sul da cidade de Curitiba, Paraná, Brasil. Cad Saude Publica, v. 25, n. 3, p. 596-604, 2009.

BRASIL. Ministério da Saúde/Organização Pan-Americana da Saúde. Guia alimentar para crianças menores de dois anos. Brasília: Ministério da Saúde/Organização Pan-Americana da Saúde, 2005. 
BRASIL. Ministério da Saúde. Secretaria de Atenção à Saúde. Departamento de Ações Programáticas e Estratégicas. II Pesquisa de Prevalência de Aleitamento Materno nas Capitais Brasileiras e Distrito Federal. Brasília: Ministério da Saúde, 2009.

BRICEÑO-LEÓN, R. Quatro modelos de integração de técnicas qualitativas e quantitativas de investigação em ciências sociais. In: GOLDENBERG, P.; MARSIGLIA, R.M.G.; GOMES, M.H. de A. (Org.). O Clássico e o Novo: tendências, objetos e abordagens em ciências sociais e saúde. Rio de Janeiro: Fiocruz, 2003. p. 157-183.

BUENO, M.B.; SOUZA, J.M.P. de; SOUZA, S.B. de et al. Riscos associados ao processo de desmame entre crianças nascidas em hospital universitário de São Paulo, entre 1998 e 1999: estudo de coorte prospectivo do primeiro ano de vida. Cad Saude Publica, v. 19, n. 5 , p. 1453-1460, 2003.

CAMilO, D.F.; CARVAlHO, R.V.B.; OliVeIRA, E.F. de et al. Prevalência da amamentação em crianças menores de dois anos vacinadas nos centros de saúde escola. Rev. Nutr, v. 17, n. 1, p. 29-36, 2004.

CAPRA, F. A teia da vida: uma nova compreensão científica dos sistemas vivos. São Paulo: Cultrix, 2006. 256 p.

CARRASCOZA, K.C.; COSTA JÚNIOR, A.L.; AMBROZANO, G.M.M. et al. Análise de variáveis biopsicossociais relacionadas ao desmame precoce. Paidéia, Ribeiräo Preto, v. 15, n. 30, p. 93-104, 2005.

CARRASCOZA, K.C.; COSTA JÚNIOR, Á.L.; AMBROZANO, G.M.M. et al. Fatores que influenciam o desmame precoce e a extensão do aleitamento materno. Estud. psicol., Campinas, v. 22, n. 4, p. 433-440, 2005.

CARVALHAES, M.A. de B.L.; PARADA, C.M.G. de L.; COSTA, M.P. da. Factors associated with exclusive breastfeeding in children under four months old in Botucatu-SP, Brazil. Rev Lat Am Enfermagem, v. 15, n. 1, p. 62-69, 2007.

CHAVES, R.G.; LAMOUNIER, J. A.; CÉSAR, C. C. Automedicação em nutrizes e sua influência sobre a duração do aleitamento materno. J Pediatr, Rio de Janeiro, v. 85, n. 2, p. 129-134, 2009.

CLARO, R.M.; BURRAJ, M.C.; SILVA, A.T. da et al. Prevalência e duração da amamentação em crianças de 0 a 2 anos na periferia de Campinas, São Paulo, Brasil, 2001. Rev. Ciênc. Méd., Campinas, v. 13, n. 4, p. 337-346, 2004.

FRANÇA, G.V.A. de; BRUNKEN, G.S.; SILVA, S.M. da et al. Determinantes da amamentação no primeiro ano de vida em Cuiabá, Mato Grosso. Rev Saude Publica, v. 41, n. 5, p. 711-718, 2007.

FROTA, D.A.L.; MARCOPITO, L.F. Amamentação entre mães adolescentes e nãoadolescentes, Montes Claros, MG. Rev Saude Publica, v. 38, n. 1, p. 85-92, 2004.

GONÇALVES, M.B.; PADULA, J.; HAYASHI, K. et al. Prevalência do aleitamento 
materno entre crianças nascidas no Hospital Universitário de Maringá no período de 19992000, Maringá, Estado do Paraná. Acta sci., Health sci, v. 25, n. 1, p. 115-124, 2003.

HASSELMANN, M.H.; WERNECK, G.L; SILVA, C.V.C. da. Symptoms of postpartum depression and early interruption of exclusive breastfeeding in the first two months of life. Cad Saude Publica, v. 24, supl.2, p. s341-s352, 2008.

LOURENÇO, M.A.; DESLANDES, S.F. Experiência do cuidado materno e amamentação sob a ótica de mulheres vítimas de violência conjugal. Rev Saude Publica, v. 42, n. 4, p. 615-621, 2008.

MACHADO, L.V.; LAROCCA, L.M. Intercorrências mamárias e desmame precoce: uma abordagem comunicacional. Cogitare enferm, v. 9, n. 2, p. 89-98, 2004.

MARTINS, P.H. MARES (Metodologia de Análise de Redes do Cotidiano): aspectos conceituais e operacionais. In: PINHEIRO, R.; MARTINS, P.H.(Org.). Avaliação em saúde na perspectiva do usuário: abordagem multicêntrica. Rio de Janeiro: Cepesc, 2009a. p. 61-89.

Repensando sociologicamente a noção linear de determinantes sociais. In: PINHEIRO, R.; MARTINS, P. H. (Org.). Avaliação em saúde na perspectiva do usuário: abordagem multicêntrica. Rio de Janeiro: Cepesc, 2009b. p. 53-60.

MASCARENHAS, M.L.W.; ALBERNAZ, E.P.; SILVA, M.B. da et al. Prevalence of exclusive breastfeeding and its determiners in the first 3 months of life in the South of Brazil. J Pediatr, Rio de Janeiro, v. 82, n. 4, p. 289-294, 2006.

MINAYO, M.C. de S. O desafio da pesquisa social. In: MINAYO, M.C. de S. (Org.). Pesquisa Social: teoria, método e criatividade. Petrópolis: Vozes, 2008a. p.9-29.

MINAYO, M. C. de S. O desafio do conhecimento: pesquisa qualitativa em saúde. São Paulo: Hucitec, 2008b.

OLIVEIRA, L.P.M. de; ASSIS, A.M.O.; GOMES, G.S. da S. et al. Duração do aleitamento materno, regime alimentar e fatores associados segundo condições de vida em Salvador, Bahia, Brasil. Cad Saude Publica, v. 21, n. 5, p. 1519-1530, 2005.

OSÓRIO, C.M.; QUEIROZ, A.B.A. Representações sociais de mulheres sobre a amamentação: teste de associação livre de idéias acerca da interrupção precoce do aleitamento materno exclusivo. Esc. Anna Nery Rev. Enferm., v. 11, n. 2, p. 261-267, 2007.

PARIZOTTO, J.; ZORZI, N.T. Aleitamento Materno: fatores que levam ao desmame precoce no município de Passo Fundo, RS. Mundo saúde (1995), v. 32, n. 4, p. 466-474, 2008. RAMOS, C.V.; ALMEIDA, J.A.G. Alegações maternas para o desmame: estudo qualitativo. J Pediatr, Rio de Janero, v. 79, n. 5, p. 385-390, 2003 a.

. Aleitamento materno: como é vivenciado por mulheres assistidas em uma unidade de referência na atenção materno-infantil em Teresina, Piauí. Rev. bras. saúde matern. infant, v. 3, n. 3, p. 315-321, 2003b. 
RAMOS, C.V.; ALMEIDA, J.A.G. de; ALBERTO, N.S.M. da C. et al. Diagnóstico da situação do aleitamento materno no Estado do Piauí, Brasil. Cad. Saude Publica, v. 24, n. 8, p. 1753-1762, 2008.

SALIBA, N.A.; ZINA, L.G.; MOIMAZ, S.A.S. et al. Freqüência e variáveis associadas ao aleitamento materno em crianças com até 12 meses de idade no município de Araçatuba, São Paulo, Brazil. Rev. bras. saúde matern. infant; v. 8, n. 4, p. 481-490, 2008.

SILVA, I.A. A vivência de amamentar para trabalhadoras e estudantes de uma universidade pública. Rev Bras Enferm, v. 58, n. 6, p. 641-646, 2005.

Situação de amamentação entre mulheres trabalhadoras e alunas de graduação e pós-graduação de uma universidade pública. Acta sci., Health sci, v. 25, n. 2, p. 215-225, 2003. SILVEIRA, F.J.F. da; LAMOUNIER, J. A. Fatores associados à duração do aleitamento materno em três municípios na região do Alto Jequitinhonha, Minas Gerais, Brasil. Cad Saude Publica, v. 22, n. 1, p. 69-77, 2006.

SOARES, M.E. de M.; GIUGLIANI, E.R. .; BRAUN, M.L. et al. Uso de chupeta e sua relação com o desmame precoce em população de crianças nascidas em Hospital Amigo da Criança. J Pediatr, Rio de Janeiro, v. 79, n. 4, p. 309-316, 2003.

TOMA, T.S.; REA, M.F. Benefícios da amamentação para a saúde da mulher e da criança: um ensaio sobre as evidências. Cad. Saúde Pública, v. 24, supl. 2, p. S235-S246, 2008.

VANNUCHI, M.T.O.; THOMSON, Z.; ESCUDER, M.M.L. et al. Perfil do aleitamento materno em menores de um ano no Município de Londrina, Paraná. Rev. bras. saúde matern. infant, v. 5, n. 2, p. 155-162, 2005.

VICTORA, C.G.; BeHAGUE, D.P.; BARROS, F.C. et al. Pacifier use and short breastfeeding duration: cause, consequence or coincidence? Pediatrics, v.99, n. 3, p. 445453, 1997.

VICTORA, C.G.; MATIJASEVICH, A.; SANTOS, I.S. et al. Breastfeeding and feeding patterns in three birth cohorts in Southern Brazil: trends and differentials. Cad Saude Publica, v. 24, supl. 3, p. S409-S416, 2008.

VIEIRA, G.O.; ALMEIDA, J.A.G. de; SILVA, L.R. et al. Fatores associados ao aleitamento materno e desmame em Feira de Santana, Bahia. Rev. bras. saúde matern. infant, v. 4, n. 2, p. 143-150, 2004.

VOLPATO, S.E.; BRAUN, A.; PEGORIM, R.M. et al. Avaliação do conhecimento da mãe em relação ao aleitamento materno durante o período pré-natal em gestantes atendidas no Ambulatório Materno Infantil em Tubarão, (SC). Arq. catarin. med., v. 38, n. 1, p. 49-55, 2009.

VOLPINI, C.C. de A.; MOURA, E.C. Determinantes do desmame precoce no distrito noroeste de Campinas. Rev. Nutr, v. 18, n. 3, p. 311-319, 2005.

WAYLAND, C. Breastfeeding patterns in Rio Branco, Acre, Brazil: a survey of reasons for weaning. Cad Saude Publica, v. 20, n. 6, p. 1757-1761, 2004. 


\section{Abstract}

Social determinants of weaning: contributions of different methodological approaches

This paper aims to study the contributions of quantitative and qualitative methods for the construction of knowledge about the social determinants of the weaning process, by comparing the findings of studies published between 2003 and 2009 in LILACS database system. The social determinants identified in quantitative research were expressed by risk variables, while those identified in the qualitative research gave visibility to inter-subjective and objective factors that shape social action. The realization that the quantity and quality are characteristics that make up the complex phenomena, leads us to the triangulation of methods as a strategy to expand the existing knowledge on social determinants of the weaning process and thereby contribute to the expansion of the breast-feeding period.

Key words: weaning; breast feeding; methodology. 